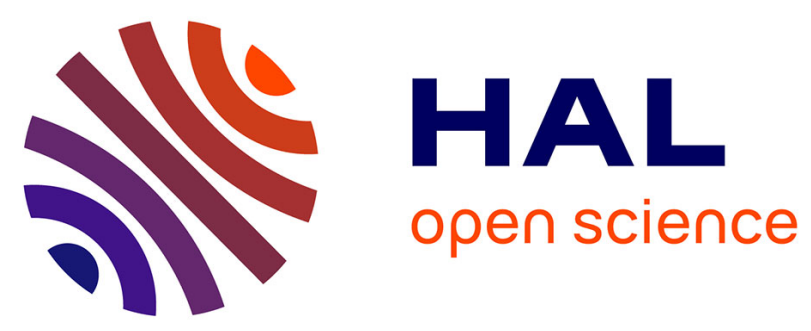

\title{
Regular Set of Representatives for Time-Constrained MSC Graphs
}

\author{
Sundararaman Akshay, Blaise Genest, Loïc Hélouët, Shaofa Yang
}

\section{To cite this version:}

Sundararaman Akshay, Blaise Genest, Loïc Hélouët, Shaofa Yang. Regular Set of Representatives for Time-Constrained MSC Graphs. [Research Report] RR-7823, INRIA. 2011, 15 p. hal-00647720

\section{HAL Id: hal-00647720 \\ https://hal.inria.fr/hal-00647720}

Submitted on 6 Dec 2011

HAL is a multi-disciplinary open access archive for the deposit and dissemination of scientific research documents, whether they are published or not. The documents may come from teaching and research institutions in France or abroad, or from public or private research centers.
L'archive ouverte pluridisciplinaire HAL, est destinée au dépôt et à la diffusion de documents scientifiques de niveau recherche, publiés ou non, émanant des établissements d'enseignement et de recherche français ou étrangers, des laboratoires publics ou privés. 


\section{N R I A}

INSTITUT NATIONAL DE RECHERCHE EN INFORMATIQUE ET EN AUTOMATIQUE

\section{Regular Set of Representatives for Time-Constrained MSC Graphs}

S. Akshay, Blaise Genest, Loïc Hélouët, Shaofa Yang

$\mathbf{N}^{\circ} \mathbf{7 8 2 3}$

November 2011

Thème COM

apport

de recherche 



\title{
RINRIA
}

\section{Regular Set of Representatives for Time-Constrained MSC Graphs}

\author{
S. Akshay, Blaise Genest, Loïc Hélouët, Shaofa Yang \\ Thème COM — Systèmes communicants \\ Équipes-Projets DISTRIBCOM - National University of Singapore
}

Rapport de recherche $\mathrm{n}^{\circ} 7823$ - November 2011 - 15 pages

Résumé : Il est notoirement difficile d'analyser les comportements de systémes décrits par des modèles qui comportent à la fois du temps et de la concurrence. Des résultats de décidabilité existent pour des modèles dans lesquels les valeurs des horloges sur différents processus ne peuvent pas être comparées, ou lorsque les modèles ont des ensembles d'exécutions temporisés réguliers. Dans ce travail, nous montrons de nouveaux résultats de décidabilité pour des modèles temporisés et concurrents, qui ne s'appuient sur aucune de ces restrictions. Nous étudions le formalisme des time-constrained MSC-graphs (TC-MSC graphs), initalement proposés par [1], et le problème qui consiste à savoir si l'ensemble des exécutions temporisées d'un modèle est vide ou non. Ce problème a été prouvé indécidable en général pour les TC-MSC graphs [10.

Notre approche pour obtenir une procédure de décision comporte deux étapes : (i) trouver un sous-ensemble $R$ d'exécutions temporisées appelé ensemble des représentants : pour toute exécution temporisée du système, on doit pouvoir trouver une exécution équivalente dans $R$ modulo commutation, (ii) prouver que $R$ est régulier. L'existence d'un ensemble de représentants régulier permet de résoudre le problème de la vacuité de l'ensemble des exécutions d'un TC-MSC graph. Nous proposons une restriction aux TC-MSC graphs, que nous appelons TC-MSC graphs bien formés. Dans un TC-MSC graph bien formé, on ne peut forcer le système à exécuter un nombre arbitrairement grand d'événements en un laps de temps fini. Il est également interdit qu'un MSC prenne obligatoirement un temps arbitrairement long pour être entièrement exécuté. Les restrictions imposées aux TC-MSC graphs bien formés réduisent peu la puissance d'expression du langage, et permettent de garantir l'existence d'un ensemble régulier de représentants.

Mots-clés : scenarios, ordres partiels, systemes distribues, automates temporisées

This work was supported by the DST INRIA associated team

This work was done when the first author was a Postdoctoral student at National University of Singapore

Centre de recherche INRIA Rennes - Bretagne Atlantique

IRISA, Campus universitaire de Beaulieu, 35042 Rennes Cedex (France)

Téléphone : +33299847100 — Télécopie : +33299847171 


\title{
Regular Set of Representatives for Time-Constrained MSC Graphs
}

\begin{abstract}
Systems involving both time and concurrency are notoriously difficult to analyze. Existing decidability results apply in settings where clocks on different processes cannot be compared or where the set of timed executions is regular. We prove new decidability results for timed concurrent systems, requiring neither restriction. We consider the formalism of time-constrained MSCgraphs (TC-MSC graphs for short) introduced in [1, and study whether the set of timed executions generated by a TC-MSC graph is empty or not. This emptiness problem is known to be undecidable in general [10.

Our approach for obtaining decidability consists of two steps: (i) find a subset $R$ of representative timed executions, that is, for which every timed execution of the system has an equivalent, up to commutation, timed execution in $R$, and (ii) prove that $R$ is regular. This allows us to solve the emptiness problem under the assumption that the TC-MSC graph $G$ is well-formed. In particular, a well-formed TC-MSC graph is prohibited from forcing any basic scenario to take an arbitrarily long time to complete. Secondly, it is forbidden from enforcing unboundedly many events to occur within a single unit of time. We argue that these restrictions are indeed practically sensible.
\end{abstract}

Key-words: Scenario languages, partial orders, distributed systems, timed automata 


\section{Introduction}

In a distributed system, several processes interact to implement a collection of global behaviors. These processes are often equipped with timing information and protocol specifications include timing requirements for messages and descriptions of how to recover from timeouts. Thus, a system designer has to deal with situations where time and concurrency influence each other. One way to describe these interactions is through scenarios, formalized using Message Sequence Charts (MSCs) [13]. The timing information is captured by adding timing constraints between pairs of events, yielding time-constrained MSCs (denoted TC-MSCs). Further, infinite collections of MSCs are typically described using High-level Message Sequence Charts, or more basic forms called MSC-graphs, i.e. directed graphs whose nodes are labelled by MSCs. MSCgraphs can be easily generalized to time-constrained MSC graphs (TC-MSC graphs) 10], whose nodes are labelled by TC-MSCs and edges have additional timing constraints. In this paper, we are interested in obtaining decidability results for the analysis of TC-MSC graphs.

Obtaining decidability in the presence of both time and concurrency is a challenging issue. In particular, even the simple question of checking if there exists a timed execution of a TC-MSC graph consistent with all the constraints is undecidable in general 10. This is the emptiness problem, which in the case of (sequential) timed automata is known to be decidable 3. Extending such decidability results to distributed systems has been done only in two particular and limited settings. In the first setting, [14, 9] consider clocks that are local to a process. But then, one cannot specify time taken by a communication (message or synchronisation). This limitation makes the specification formalism very weak. The second setting can relate clocks from different processes and specify how long a communication can or must take [1, 2, 6, 7]. However, these papers restrict the concurrency in a structural way, for instance considering only locally synchronized (see [16, 4, 12]) MSC-graphs (in [1, 2]) or only safe Petri Nets (in 6, 7]). The language of the specification is then forced to be regular, which is a significant restriction in a concurrent setting where even simple behaviors may not be regular (e.g., the producer-consumer protocol).

In this paper, we propose the first decidability result for timed concurrent systems with global clocks having a possibly non-regular set of behaviors. More specifically, we tackle the emptiness problem for TC-MSC graphs (which is undecidable in general [10]) by coming up with mild restrictions which are practically motivated and yet sufficient to prove decidability. Indeed, this is important as the only non trivial way to have an empty language is that every possible timing of the events conflict with some constraint. Such specification should then be considered as ill-formed, which has to be reported to the designer.

Our technique to obtain decidability of the emptiness problem is to use a regular set of representatives. A set of representatives is a subset of executions such that every execution of the system has an equivalent- up to commutationexecution in this subset. This technique has been used previously in untimed settings [15, 11, 8] and with the fixed set of existentially bounded executions [11] as the regular set of representatives. In Section 3, it is formalized as a general technique on timed languages, and is applied on a new regular set of representatives called the set of well-behaved timed executions. This is the subset of timed executions where two events from the same scenario do not occur 
at dates that are arbitrarily apart from each other (non drifting), and where there are a limited number of events done in one unit of time (non Zeno). This demonstrates the versatility of this technique.

We state our main theorems in Section 4 : the set of well-behaved timed executions is regular, and it is a set of representatives under the assumption that the TC-MSC graph is well-formed. Together, these imply that the emptiness problem is decidable for well-formed TC-MSC graphs. Intuitively, being wellformed forbids specifications in which (1) events from the same scenario are forced to occur arbitrarily apart from each other (drifting), which is undesirable as it goes against the MSC-graph design, and (2) an unbounded number of events are forced to happen within one unit of time, which is unimplementable.

The proofs of these Theorems are detailed in Section 5 . The regularity of the set of well-behaved executions exploits the fact that if node $x$ appears sufficiently before node $y$ in a path, then all events of $x$ must occur before any event of $y$ in any well-behaved execution of this path. Proving representativity for a wellformed TC-MSC graph is non trivial, as for each execution, one needs to find a representative which is both non drifting and has a limited number of events per unit of time, while being well-formed guarantees only the existence of two representatives, one of each kind. Finally, we discuss in Section 6 the significance and practicality of our assumptions as well as related work.

\section{Time-Constrained MSC graphs}

We begin by fixing a finite nonempty set of processes $\mathcal{P}$ that communicate through messages via reliable FIFO channels. Let $p, q$ range over $\mathcal{P}$. The communication alphabet is given by $\Sigma=\{p ! q, p ? q \mid p \neq q\}$ where the sending action $p ! q$ denotes a message sent from process $p$ to $q$ and the receiving action $q ? p$ denotes a message received by process $q$ from $p$. Let $\mathbb{N}$ denote the set of natural numbers. Further, let $\mathcal{I}(\mathbb{N})$ denote the set of open and closed intervals whose end points are in $\mathbb{N}$, plus the intervals of the form $[c, \infty),(c, \infty)$, where $c \in \mathbb{N}$. We shall use intervals in $\mathcal{I}(\mathbb{N})$ to constrain the lower and upper bounds on the difference of occurrence times of events in a scenario. Note that intervals involving non-negative rationals can be easily simulated by scaling them to integers. We adopt the basic definitions from [1].

Definition 2.1. A time-constrained message sequence chart (TC-MSC) over $\mathcal{P}$ and $\Sigma$ is a tuple $T=\left(E,\left(<_{p}\right)_{p \in \mathcal{P}}, \lambda, \mu, \delta\right)$ where $E$ is a finite nonempty set of events $; \lambda: E \rightarrow \Sigma$ labels each event with a letter in $\Sigma$. The following conditions are satisfied :

- Each $<_{p} \subseteq E_{p} \times E_{p}$ is a total order, where $E_{p}=\lambda^{-1}(\{p\} \times\{!, ?\} \times \mathcal{P})$. Members of $E_{p}$ are termed p-events.

- The message relation $\mu$ is a bijection from $E_{\text {send }}=\lambda^{-1}(\mathcal{P} \times\{!\} \times \mathcal{P})$ (the sending events) to $E_{\text {recv }}=\lambda^{-1}(\mathcal{P} \times\{?\} \times \mathcal{P})$ (the receiving events). For any $e, f$ with $\mu(e)=f$, it is the case that for some $p, q$, we have $\lambda(e)=p ! q$ and $\lambda(f)=q$ ? $p$. For each $p$, any events $e, e^{\prime}$ in $E_{p}$, satisfies that $e<_{p} e^{\prime}$ iff $\mu(e)<_{q} \mu\left(e^{\prime}\right)$, where $\mu(e)$ is a q-event.

- Write $<$ for the transitive closure of $\left(\bigcup_{p \in \mathcal{P}}<_{p}\right) \cup \mu$. The time constraint labelling function $\delta$ associates an interval in $\mathcal{I}(\mathbb{N})$ to each pair of events $(e, f) \in E \times E$ with $e<f$. 
Abusing notation, we write a TC-MSC more conveniently as $(E,<, \lambda, \mu, \delta)$, with the understanding that $<$ is in fact defined from $<_{p}$ for $p$ ranging over $\mathcal{P}$, and $\mu$. In what follows, we fix a TC-MSC $T=(E,<, \lambda, \mu, \delta)$.

A linearization of $T$ is a sequence $\sigma=a_{1} \ldots a_{\ell}$ over $\Sigma^{*}$, where $\ell=|E|$ and such that $E$ can be enumerated as $e_{1} \ldots e_{\ell}$ with $a_{i}=\lambda\left(e_{i}\right)$, and $e_{i}<e_{j}$ implies $i<j$ for any $i, j$ in $\{1, \ldots, \ell\}$. Note that due to the FIFO condition, the enumeration $e_{1} \ldots e_{\ell}$ is uniquely determined by $a_{1} \ldots a_{\ell}$. A TC-MSC $T$ defines a collection of linearizations augmented with occurrence times such that the relative delay of each pair of causally ordered event falls within the interval dictated by $\delta$. To avoid confusion, we shall term occurrence times as dates :

Definition 2.2. Let $T$ be as fixed above. A timed execution $w$ of $T$ is a sequence $\left(a_{1}, d_{1}\right) \ldots\left(a_{\ell}, d_{\ell}\right)$, where $a_{1} \ldots a_{\ell}$ is a linearization of $T$, each date $d_{i}$ is a nonnegative real for $i=1, \ldots, \ell$, and $d_{1} \leq \ldots \leq d_{\ell}$. Let $e_{1} \cdots e_{\ell}$ be the enumeration corresponding to the linearization $a_{1} \ldots a_{\ell}$. Then $e_{i}<e_{j}$ implies $d_{j}-d_{i}$ is in the interval $\delta\left(e_{i}, e_{j}\right)$.

To describe infinite collections of TC-MSCs, one uses the formalism of TCMSC graphs.

Definition 2.3. Let $\mathcal{T}$ be a finite nonempty set of TC-MSCs. A TC-MSC graph over $\mathcal{T}$ is a tuple $G=\left(N, \longrightarrow, n_{\text {ini }}, N_{\text {fin }}, \Lambda, \Delta\right)$ where $N$ is a finite set of nodes, $\longrightarrow \subseteq N \times N$ a transition relation, $n_{\text {ini }} \in N$ the initial node, $N_{\text {fin }} \subseteq N$ the subset of final nodes, and $\Lambda: N \rightarrow \mathcal{T}$ labels each node with a TC-MSC from $\mathcal{T}$. Further, the mapping $\Delta$ associates each transition $\left(n, n^{\prime}\right)$ in $\longrightarrow$ with a $\mathcal{P}$-indexed family of intervals in $\mathcal{I}(\mathbb{N})$.

For each $p$, we write $\Delta_{p}\left(n, n^{\prime}\right)$ for the $p$-th component of $\Delta\left(n, n^{\prime}\right)$. The interval $\Delta_{p}\left(n, n^{\prime}\right)$ specifies the range of relative delay on $p$ when moving from $n$ to $n^{\prime}$. We write $\perp$ for the interval $[0, \infty)$. Figure 1 displays a TC-MSC graph whose nodes consist of $n_{1}, n_{2}, n_{3}$. The initial node $n_{1}$ is indicated by an incoming arrow. There is only one final node, $n_{3}$. In node $n_{1}$, the relative delay between the sending event of $p$ and the receiving event of $q$ is constrained to lie within $[0,3]$. The $([0,2], \perp, \perp)$ on transition $\left(n_{1}, n_{2}\right)$ indicates $\Delta_{p}\left(n_{1}, n_{2}\right)=[0,2]$, $\Delta_{q}\left(n_{1}, n_{2}\right)=\perp, \Delta_{r}\left(n_{1}, n_{2}\right)=\perp$. It asserts that the relative delay between the last event of $p$ of $n_{1}$ and the first event of $p$ of $n_{2}$ should be in [0,2]. To reduce clutter in the figures, we shall omit all time constraints of $\perp$ inside a basic scenario.

We fix a TC-MSC graph $G=\left(N, \longrightarrow, n_{\text {ini }}, N_{\text {fin }}, \Lambda, \Delta\right)$. We write $n \longrightarrow n^{\prime}$ for $\left(n, n^{\prime}\right) \in \longrightarrow$. We shall speak interchangeably of a node $n$ and its associated TC-MSC $\Lambda(n)$. A TC-MSC graph defines a collection of TC-MSCs arising from concatenating TC-MSCs in paths of $G$. We first define this mechanism of concatenating adjacent TC-MSCs in $G$. For a TC-MSC $T=(E,<, \lambda, \mu, \delta)$, we call the $<_{p}$-minimal event in $E_{p}$ the first $p$-event, and the $<_{p}$-maximal event in $E_{p}$ the last $p$-event. Simply put, for a transition $\left(n, n^{\prime}\right)$, the concatenation of $n$ with $n^{\prime}$ is the TC-MSC resulting from placing $n^{\prime}$ after $n$, and for each process $p$, take $\Delta_{p}\left(n, n^{\prime}\right)$ to be the time constraint between the last $p$-event of $n$ and the first $p$-event of $n^{\prime}$.

Definition 2.4. Let $G$ be as fixed above and $\left(n, n^{\prime}\right)$ a transition of $G$. Let $\Lambda(n)=(E,<, \lambda, \mu, \delta)$ and $\Lambda\left(n^{\prime}\right)=\left(E^{\prime},<^{\prime}, \lambda^{\prime}, \mu^{\prime}, \delta^{\prime}\right)$. The concatenation of $\Lambda(n)$ and $\Lambda\left(n^{\prime}\right)$, denoted $\Lambda(n) \circ \Lambda\left(n^{\prime}\right)$, is the $T C-M S C\left(E^{\prime \prime},<^{\prime \prime}, \lambda^{\prime \prime}, \mu^{\prime \prime}, \delta^{\prime \prime}\right)$ detailed as 

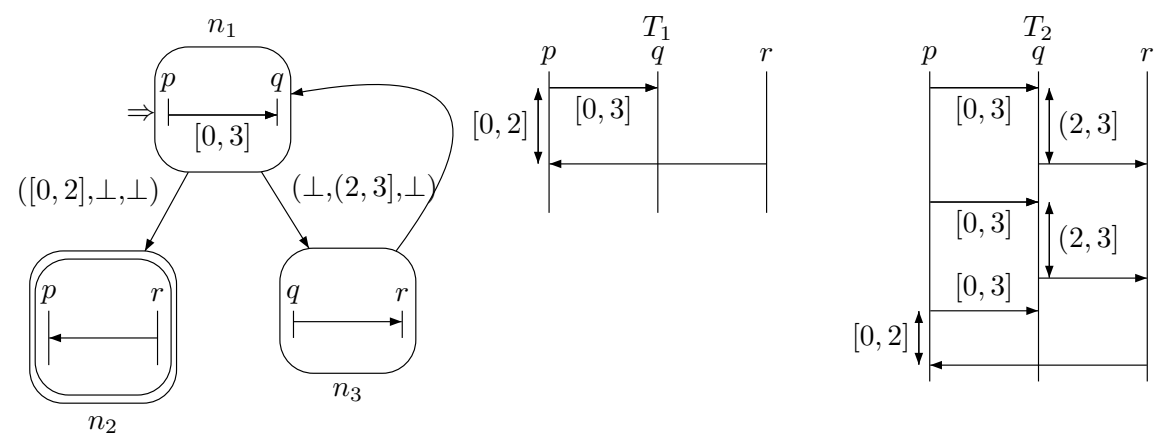

Figure 1 - A TC-MSC graph $G_{1}$ and two TC-MSCs it generates

follows. Firstly, $E^{\prime \prime}$ is the disjoint union of $E$ and $E^{\prime} ; \lambda^{\prime \prime}$ agrees with $\lambda$ on events in $E$, and with $\lambda^{\prime}$ on events in $E^{\prime}$. Secondly, for each $p,<_{p}^{\prime \prime}$ is $<_{p} \cup<_{p}^{\prime} \cup E_{p} \times E_{p}^{\prime}$; $\mu^{\prime \prime}$ is the union of $\mu$ and $\mu^{\prime}$. Lastly, for $e, f \in E^{\prime \prime}$ with $e<^{\prime \prime} f, \delta^{\prime \prime}(e, f)$ is given as follows : (i) if $e, f \in E$, then $\delta^{\prime \prime}(e, f)=\delta(e, f)$; (ii) if $e, f \in E^{\prime}$, then $\delta^{\prime \prime}(e, f)=\delta^{\prime}(e, f)$; (iii) suppose $e \in E, f \in E^{\prime}$. If for some $p$, $e$ is the last $p$-event of $n$ and $f$ the first $p$-event of $n^{\prime}, \delta^{\prime \prime}(e, f)=\Delta_{p}\left(n, n^{\prime}\right)$, otherwise, $\delta(e, f)=\perp$.

Note that as in 1, 10, 2, in the above definition, if either $E_{p}=\emptyset$ or $E_{p}^{\prime}=\emptyset$, then $\Delta_{p}\left(n, n^{\prime}\right)$ is disregarded in $\Lambda(n) \circ \Lambda\left(n^{\prime}\right)$. That is, we can assume without loss of generality that $\Delta_{p}=\perp$ for each such $p$ on such a transition $\left(n, n^{\prime}\right)$. Observe that now $\circ$ is associative.

A path of $G$ is a sequence of nodes $\rho=n_{0} \ldots n_{\ell}$ of $G$ such that each $n_{0}=n_{\text {ini }}$ and $n_{i} \longrightarrow n_{i+1}$ for $i=0, \ldots, \ell-1$. We emphasize that a path always starts with the initial node. Since $\circ$ is associative, we can unambiguously define the TC-MSC induced by $\rho$, denoted $T^{\rho}$, to be $\Lambda\left(n_{0}\right) \circ \ldots \circ \Lambda\left(n_{\ell}\right)$. A path is final if its last node is in $N_{f i n}$.

The TC-MSC language of $G$ is the set of TC-MSCs induced by final paths of $G$. For a TC-MSC $T$, let $L(T)$ denote its set of timed executions. For the TC-MSC graph $G$, the timed execution language of $G$, denoted $L(G)$, is the union of $L\left(T^{\rho}\right)$ ranging over final paths $\rho$ of $G$. We say that a TC-MSC $T$ (resp. a path $\rho)$ is consistent iff $L(T) \neq \emptyset\left(\right.$ resp. $\left.L\left(T^{\rho}\right) \neq \emptyset\right)$.

We tackle the emptiness problem of TC-MSC graphs, which can be stated as : given a TC-MSC graph $G$, determine whether $L(G)$ is empty. The emptiness of $L(G)$ implies that for any TC-MSC $T^{\rho}$ induced by a final path $\rho$ of $G$, no assignment of dates to events in $T^{\rho}$ can satisfy all the time constraints in $T^{\rho}$. Thus, such a $G$ with $L(G)=\emptyset$ should be considered ill-specified, and thus it should be checked for. However, it is known [10] that :

Proposition 2.1. [10] The emptiness problem of TC-MSC graphs is undecidable.

In [1, 2], decidability is obtained for locally-synchronized TC-MSC graphs. This syntactical restriction limits concurrency, and implies that the timed execution language is regular, which is a severe restriction. Indeed, even simple examples, such as $G_{1}$ from Figure 1 or the producer-consumer protocol, do not have regular timed execution languages. 

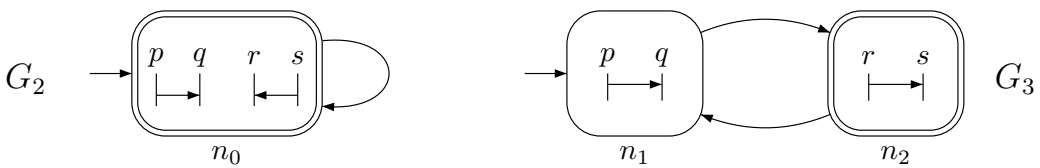

Figure 2 - Two TC-MSC graphs $G_{2}, G_{3}$. Specification $G_{2}$ is scenario-connected and $G_{3}$ is not.

\section{$3 \quad$ Regular Set of Representatives}

We advocate a technique of using regular sets of representatives for obtaining decidability of the emptiness problem of TC-MSC graphs. This is a partial order reduction technique (since not all timed executions will be considered), which can handle TC-MSC graphswith non-regular timed execution languages. We begin with the following definition :

Definition 3.1. Let $G$ be a TC-MSC graph. A subset $R$ of $L(G)$ is called a set of representatives for $G$ if for each consistent final path $\rho$ of $G, R \cap L\left(T^{\rho}\right) \neq \emptyset$.

It immediately follows that :

Proposition 3.1. If $R$ is a set of representatives for $G$, then $L(G)=\emptyset$ iff $R=\emptyset$.

Indeed, many timed executions of a TC-MSC graph $G$ are equivalent, in the sense that they are timed executions of the TC-MSC induced by the same final path of $G$. To check for emptiness of $L(G)$, it suffices to consider emptiness of a set $R$ of representatives for $G$, instead of $L(G)$ itself. If $R$ turns out to be regular and effective, then the emptiness problem of TC-MSC graphs can be decided. For example, consider $G_{2}$ in Figure 2. The language $L\left(G_{2}\right)$ is not regular. However, the set $\left\{\sigma_{0}, \sigma_{0} \sigma_{1}, \sigma_{0} \sigma_{1} \sigma_{2}, \ldots\right\}$, where $\sigma_{i}=(p ! q, 4 i)(q ? p, 4 i+$ 1) $(s ! r, 4 i+2)(r ? s, 4 i+3)$ for all $i \in \mathbb{N}$, is a regular set of representatives for $G_{2}$.

Thus, there are three elements in the technique of regular set of representatives :

1. Choose a subset $R$ of $L(G)$.

2. Show that the chosen subset $R$ is a set of representatives for $G$.

3. Prove that $R$ is regular.

Till the end of the section, let us fix a TC-MSC graph $G=\left(N, \longrightarrow, n_{i n i}, N_{f i n}, \Lambda, \Delta\right)$, a path $\rho=n_{0} \ldots n_{\ell}$ of $G$, a timed execution $w=\left(a_{1}, d_{1}\right) \ldots\left(a_{h}, d_{h}\right)$ of $\rho$, and $e_{1}, \cdots e_{h}$ the enumeration of $E$ associated with $a_{1} \cdots a_{h}$. We start by giving a first set of representatives.

Definition 3.2. Let $K$ be an integer. We say that $w$ is $K$-drift-bounded if for each $0 \leq u \leq \ell$, and $i, j \in\{1, \ldots, h\}$, if $e_{i}, e_{j}$ are in $\Lambda\left(n_{u}\right)$, then $\left|d_{i}-d_{j}\right| \leq K$.

In other words, $w$ is $K$-drift-bounded if the difference between the first and the last date associated with an event of any basic scenario is bounded by $K$. Interpreting the scenario in each node of a TC-MSC graph as one phase or one transaction of a distributed protocol, it is realistic to believe that exectuions of an implemented system are $K$-drift-bounded. 
Now, for a TC-MSC graph $G$ and an integer $K$, we say that $G$ is $K$-driftbounded if for every consistent path $\rho$ of $G$, there exists a $K$-drift-bounded timed execution in $L(\rho)$. We emphasize that all timed executions of $L(\rho)$ are not required to be $K$-drift-bounded. Observe that with the above definitions, $G$ being $K$-drift bounded implies that the set $L_{K}(G)$ of $K$-drift-bounded executions of $G$ is a set of representatives of $G$. Unfortunately this set may not be regular. For example, for the TC-MSC graph $G_{2}$ in Figure 2, $L_{K}\left(G_{2}\right)$ is not regular, for any $K$, because of timed executions with an unbounded number of events per unit of time. Formally, for an integer $K^{\prime}$, we say that $w$ has at most $K^{\prime}$ events per unit of time if for any $i, j$ in $\{1, \ldots, h\}, d_{j}-d_{i} \leq 1$ implies $j-i<K^{\prime}$.

This phenomenon of having unboundedly many events within one unit of time is known as Zenoness and occurs commonly in timed specifications. It turns out that by imposing the next simple syntactical condition, one can prevent a TC-MSC graph from enforcing Zenoness (this is one consequence of Theorem 4.2 below). We say that a transition $\left(n, n^{\prime}\right)$ of $G$ is positively constrained if for every $p, \Delta_{p}\left(n, n^{\prime}\right)$ is not $[0,0]$ (but it can be $[0,1),[3,3],[2, \infty) \ldots$ ). We say that $G$ is positively constrained if every transition of $G$ is positively constrained. We can now present our regular set of representatives, namely the set $L_{K, K^{\prime}}(G)$ of $\left(K, K^{\prime}\right)$-well-behaved timed executions, defined as follows :

Definition 3.3. For integers $K, K^{\prime}$, we say that $w$ is $\left(K, K^{\prime}\right)$-well-behaved if $w$ is $K$-drift-bounded and has at most $K^{\prime}$ events per unit of time.

To get the representativity of $L_{K, K^{\prime}}(G)$, we need a restriction on the TCMSC graph :

Definition 3.4. We say that a TC-MSC graph is $K$-well-formed if it is $K$ drift-bounded and positively constrained.

\section{Main results :}

We can now state our main results. The first two theorems below hold with one more technical restriction imposed on TC-MSC graphs. However, the third theorem will establish decidability of the emptiness problem of TC-MSC graphs even without this technical restriction. A transition $\left(n, n^{\prime}\right)$ of $G$ is said to be scenario-connected if there exists a process $p$, s.t. both $n$ and $n^{\prime}$ have at least one $p$-event. $G$ is scenario-connected if every transition of $G$ is scenario-connected. For instance, in Figure 2, $G_{2}$ is scenario-connected while $G_{3}$ is not.

Theorem 4.1. Let $K, K^{\prime}$ be integers. If $G$ is scenario-connected, then $L_{K, K^{\prime}}(G)$ is regular.

Concerning the representativity, we need $G$ to be well-formed.

Theorem 4.2. Let $K$ be an integer. If $G$ is $K$-well-formed and scenarioconnected, then $L_{K, K^{\prime}}(G)$ is a set of representatives of $G$, with $K^{\prime}=4(|\mathcal{P}|+$ $1)^{2} \cdot K \cdot M$, where $M$ is the maximal number of events in one node of $G$.

Theorems 4.14 .2 implies, with effort to lift the scenario-connected assumption :

Theorem 4.3. Given a $K$-well-formed $T C$-MSC graph $G$ for some integer $K$, it is decidable to determine whether $L(G)=\emptyset$. 
An immediate question is if given a TC-MSC graph $G$ and an integer $K$, one can effectively determine whether $G$ is $K$-well-formed. In fact, it turns out that this question is decidable. However, this problem is not in the scope of this paper, and its proof, involving vastly different techniques, will be dealt with in a subsequent paper.

\section{Proofs of Representativity, Regularity and De- cidability}

\section{$5.1 \quad$ Regularity}

We prove Theorem 4.1 by constructing a timed automaton ([3]) $\mathcal{A}$ which recognizes $L_{K, K^{\prime}}(G)$. In general, to recognize $L(G)$, a timed automaton $\mathcal{A}$ needs to generate every linearization of the TC-MSC induced by every path of $G$. In linearizing events from the TC-MSC induced by a path $\rho=n_{0} \ldots n_{z}$, the discrete state of $\mathcal{A}$ keeps track of nodes which have not yet been (fully) executed, and for each such node memorizes which events have yet to be executed [16. To ensure that time constraints are respected when linearizing $\rho, \mathcal{A}$ needs to draw clocks from a fixed pool to keep track of the relative delay between an executed event $e$ and an event $f$ not yet executed, when $e, f$ are in the same node or when $e$ is the last $p$-event of $n_{i}$ and $f$ is the first $p$-event of $n_{i+1}$ [1]. In general, this requires memorizing an arbitrarily large number of events, and it requires an infinite pool of clocks.

However, to recognize $L_{K, K^{\prime}}(G)$, it suffices to remember a finite number of nodes and events, which follows from the following crucial property. Notice that bounding the number of nodes and events also bounds the set of clocks needed [1]. If $\rho=n_{0} \ldots n_{z}$ is the current path (that is, at least one event of $n_{z}$ has been executed), then the not yet (fully) executed nodes are in the suffix $n_{y} \ldots n_{z}$ of $\rho$, where $z-y$ is bounded by a fixed constant $C$ depending only on $|\mathcal{P}|, K, K^{\prime}$. This property is proved formally in Lemma 5.1. Once this is established, the detailed construction of $\mathcal{A}$, which is sketched above, follows precisely [1] (see also [2]). The main difference is that in [1, 2], the bound $C$ is obtained by restricting the concurrency.

Before stating the lemma, let us fix some notations. Let $G, K, K^{\prime}$ be as given in theorem 4.1. Let $C_{K, K^{\prime}}=2 \cdot|\mathcal{P}| \cdot(|\mathcal{P}|+1) \cdot K \cdot K^{\prime}$ and $\rho=n_{0} \ldots n_{z}$ be a path of $G$. Let $w=\left(a_{1}, d_{1}\right) \ldots\left(a_{\ell}, d_{\ell}\right)$ be a $\left(K, K^{\prime}\right)$-well-behaved timed execution of $\rho$. We denote by $e_{1}, \ldots, e_{\ell}$ the enumeration of events of $T^{\rho}$ associated with $a_{1}, \ldots, a_{\ell}$. For all $i \leq \ell$, we define $d\left(e_{i}\right)=d_{i}$.

Lemma 5.1. Let $n_{x}$ be a node of the path $\rho=n_{0} \ldots n_{z}$ given above, and such that $z-x \geq C_{K, K^{\prime}}$. Then $d(e)<d(f)$ for all events $e$ of $n_{x}$ and $f$ of $n_{z}$.

Proof. Let Events $s_{x}$ be the collection of events in the suffix $n_{x} \ldots n_{z}$. Assume that $z-x$ is sufficiently large. From the fact that $w$ has at most $K^{\prime}$ events per unit of time, we first prove that there exist two events $g, g^{\prime}$ in Events Ev $_{x}$ such that $d\left(g^{\prime}\right)>d(g)+2 C_{1}$, for some large $C_{1}$. Note that each node of $\rho$ contains at least one event. As $z-x$ is sufficiently large, there are $m \geq 2 K^{\prime} \times C_{1}$ events $f_{1}<_{p} \ldots<_{p} f_{m}$ in Events $x$ and on the same process $p$, for some $p \in \mathcal{P}$. Since $w$ has at most $K^{\prime}$ events per unit of time, it follows that $d\left(f_{m}\right)>d\left(f_{1}\right)+2 C_{1}$. 
Consider the event $g$ built as above, and let $n_{s}$ be the state it is in. We will prove that $d(g) \geq d(e)-|\mathcal{P}| \cdot K$ (actually this is true for all events $g$ in Events $x$ ). Since $G$ is scenario-connected, it follows that for each node $n_{t}$ of $\rho$, there exists a process $p_{t}$, an event $f_{t}$ in node $n_{t}$ and an event $g_{t}$ in node $n_{t+1}$ such that both $f_{t}, g_{t}$ are on porcess $p_{t}$. There exists a subsequence of $\left(f_{t}, g_{t}\right)_{0 \leq t \leq z}$ consisting of $|\mathcal{P}|$ pairs $\left(f_{i}^{\prime}, g_{i}^{\prime}\right)_{i \leq|\mathcal{P}|}$ s.t. $f_{1}$ and $e$ are in the same node, $f_{i+1}^{\prime}$ and $g_{i}^{\prime}$ are in the same node for each $i<|\mathcal{P}|$ and $g$ and $g_{|\mathcal{P}|}^{\prime}$ are in the same node. To obtain this, we only ensure that $f_{i}^{\prime}$ and $f_{j}^{\prime}$ are on different processes for all $i \neq j$.

First, we have $f_{i}^{\prime}<g_{i}^{\prime}$ for each $i \leq|\mathcal{P}|$, as they are on the same process. Hence $d\left(f_{i}^{\prime}\right) \leq d\left(g_{i}^{\prime}\right)$. Also, $\left|d(e)-d\left(f_{1}^{\prime}\right)\right| \leq K$ since $e, f_{1}^{\prime}$ are in the same node. Similarly, $\left|d\left(f_{i+1}^{\prime}\right)-d\left(g_{i}^{\prime}\right)\right| \leq K$ for each $i \leq|\mathcal{P}|$, and $\left|d\left(g_{u}^{\prime}\right)-d(g)\right| \leq K$. Thus, $d(g) \geq d(e)-(|\mathcal{P}|+1) \cdot K$. The same analysis gives $d(f) \geq d\left(g^{\prime}\right)-(|\mathcal{P}|+1) \cdot K$. Taking $C_{1}=(|\mathcal{P}|+1) \cdot K$, and recalling that $d\left(g^{\prime}\right)>d(g)+2 C_{1}$, we get $d(f)>d(e)$.

\subsection{Representativity}

Secondly, we prove theorem 4.2. Let $\rho=n_{0} \ldots n_{z}$ be a consistent path of $G$. As $G$ is $K$ drift bounded, there exists a $K$-drift-bounded timed execution $w=\left(a_{1}, d_{1}\right) \ldots\left(a_{\ell}, d_{\ell}\right)$ of $T^{\rho}$. We construct another timed execution $w^{\prime}=\left(a_{1}, d_{1}^{\prime}\right) \ldots\left(a_{\ell}, d_{\ell}^{\prime}\right)$ from $w$ by suitably modifying the dates, such that $w^{\prime}$ is still an execution of $T^{\rho}$, is $K$-drift-bounded, and has at most $K^{\prime}$ events per unit of time, for a suitable choice of $K^{\prime}$. The key idea in the construction of $w^{\prime}$ is to inductively postpone (when needed/possible) the dates of all events of $n_{x} \cdots n_{z}$. By postponing, we ensure that there will exist some process $p$ such that the difference between the date of the last $p$-event of $n_{x-1}$ and the date of the first $p$-event of $n_{x}$ is at least $1 / 2$. If it is already the case, then we do not postpone.

As before, let $e_{1} \ldots e_{\ell}$ be an enumeration of events in $T^{\rho}$ corresponding to $a_{1} \ldots a_{\ell}$, and let us write $d(e)\left(\operatorname{resp} . d^{\prime}(e)\right)$ for the date $d_{i}\left(\operatorname{resp} d_{i}^{\prime}\right)$, when $e=e_{i}$. To construct $w^{\prime}$, we first initialize $d^{\prime}(e)=d(e)$ for each event $e$. Next, consider node $n_{1}$. Let $Q \neq \emptyset$ be the collection of those processes $p$ such that both $n_{0}$ and $n_{1}$ have $p$-events. For each $p$ in $Q$, let $l e_{p}\left(n_{0}\right)$ denote the last $p$-event of $n_{0}$ and $f e_{p}\left(n_{1}\right)$ denote the first $p$-event of $n_{1}$.

If for some $p \in Q, d\left(f e_{p}\left(n_{1}\right)\right)-d\left(l e_{p}\left(n_{0}\right)\right) \geq 1 / 2$, then for each event $e$ in $n_{1}$, do not modify $d^{\prime}(e)$ (it will not be modified later either). Otherwise, let $\theta_{\max }<1 / 2$ be the maxium of $d\left(f e_{p}\left(n_{1}\right)\right)-d\left(l e_{p}\left(n_{0}\right)\right)$ where $p$ ranges over $Q$. For each event $e$ in $n_{1} \ldots n_{z}$, set $d^{\prime}(e)=d(e)+1 / 2-\theta_{\max }$. We emphasize that when considering node $n_{1}$, the above procedure postpones dates of events in $n_{1}$, and dates of events in $n_{2} \ldots n_{z}$, by the same amount. Since $G$ is positively constrained, the timed execution resulting from the above procedure is still in $L\left(T^{\rho}\right)$ and is still $K$-drift-bounded. We inductively carry on the above procedure to consider each of the nodes $n_{2}, \ldots, n_{z}$. The timed execution $w^{\prime}$ is obtained after considering all the nodes $n_{0}, \ldots, n_{z}$. It follows that $w^{\prime}$ is $K$-drift-bounded and is in $L(\rho)$.

It remains to show that $w^{\prime}$ has at most $K^{\prime}$ events per unit of time, for a sufficiently large $K^{\prime}$. Let $M$ be the maximum number of events in any node of G. It suffices to show that every pair of events $e, f$ from two nodes $n, n^{\prime}$ sufficiently apart in the path (and $n$ appearing first) satisfies $d^{\prime}(f)>d^{\prime}(e)+1$, as then events in the same unit of time belong to nodes sufficiently close, and there are at most ( $M$ times the number of nodes) such events. The proof follows 
on the exact same lines as the regularity proof above, with the same constant $C_{1}=(|\mathcal{P}|+1) K$. We show that we can find two events $g, g^{\prime}$ on nodes between $n, n^{\prime}$ with $d^{\prime}\left(g^{\prime}\right)>d^{\prime}(g)+2 C_{1}+1$. The regularity proof showed that fact using the bounded number of events per unit of time of $w$. Here, this is what we want to prove, so we rely instead on the construction of $w^{\prime}$ above. If there are more than $|\mathcal{P}| \cdot\left(4 C_{1}+2\right)$ nodes between $n$ and $n^{\prime}$, then there is one process $p$ which is in $Q$ for more than $4 C_{1}+2$ transitions. By construction of $w^{\prime}$, on each of these transitions, time elapses by $1 / 2$ on $p$, and the result follows. Now, we can apply the results from the regularity proof, to get $d^{\prime}(f)>d^{\prime}\left(g^{\prime}\right)+C_{1}$ and $d^{\prime}(g)>d^{\prime}(e)+C_{1}$, which gives $d^{\prime}(f)>d^{\prime}(e)+1$. That is, $K^{\prime}=|\mathcal{P}| \cdot M \cdot\left(4 C_{1}+2\right)$ is sufficient.

\subsection{Decidability}

From theorem 4.1 and 4.2 , one readily concludes :

Corollary 5.2. Let $G$ be a TC-MSC graph as fixed earlier, and $K$ an integer. If $G$ is scenario-connected, and $K$-well-formed, then one can effectively determine whether $L(G)$ is empty.

We now lift the scenario-connected restriction, proving Theorem 4.3. Suppose $G$ is not scenario-connected. Let $N S C$ denote the set of transitions of $G$ that are not scenario-connected. Proposition 5.3 states the crucial observation that for any path $\rho=n_{0} \cdots n_{\ell}$ with $\left(n_{i}, n_{i+1}\right)$ in $N S C$, the dates of events in $n_{i+1} \ldots n_{\ell}$ are not constrained in any way by the dates of events in $n_{0} \cdots n_{i}$. This fact was also used in [10] along the same lines.

Proposition 5.3. Suppose $\rho=n_{0} \cdots n_{\ell}$ is a path of $G$.

- If $\left(n_{i}, n_{i+1}\right)$ is in NSC where $0 \leq i<\ell$, then $\rho$ is consistent iff both path $n_{0} \cdots n_{i}$ and path $n_{i+1} \cdots n_{\ell}$ are consistent.

- Suppose $\rho$ is consistent. If $\left(n_{i}, n_{i+1}\right),\left(n_{j}, n_{j+1}\right)$ are both in NSC where $0 \leq i<j<\ell$, and $n_{i}=n_{j}$, then the path $n_{0} \ldots n_{i} n_{j+1} \ldots n_{\ell}$ is also consistent.

We now decompose $G$ into a finite collection $\mathcal{H}$ of TC-MSC graphs, each of which is scenario-connected. We will decide the non emptiness of $L(G)$ by considering the non emptiness of $L(H)$ for every $H$ in $\mathcal{H}$, which is decidable by Corollary 5.2 Let $N_{1}$ be the subset of nodes $n$ of $G$ such that $\left(n^{\prime}, n\right) \in N S C$ for some node $n^{\prime}$ of $G$. Let $N_{2}$ be the subset of nodes $n^{\prime}$ of $G$ such that $\left(n^{\prime}, n\right) \in N S C$ for some node $n$ of $G$. For each $n \in N_{1} \cup\left\{n_{i n i}\right\}$, each $n^{\prime} \in N_{2} \cup N_{\text {fin }}$, we build the scenario-connected TC-MSC graph $H_{n, n^{\prime}}$ from $G$ as follows. The set of nodes of $H_{n, n^{\prime}}$ is the same as $G . H_{n, n^{\prime}}$ has $n$ as initial node, and has one single final node $n^{\prime}$. The transitions of $H_{n, n^{\prime}}$ consist of all scenario-connected transitions of $G$. Let $\mathcal{H}$ be the collection of all such $H_{n, n^{\prime}}$. For each $H_{n, n^{\prime}}$ in $\mathcal{H}$, we can decide whether $L\left(H_{n, n^{\prime}}\right)$ is not empty following Corollary 5.2. From the first item of Proposition 5.3, $L(G)$ is not empty iff there exist a sequence $H_{n_{0}, n_{1}}$, $H_{n_{2}, n_{3}}, \ldots, H_{n_{2 \ell}, n_{2 \ell+1}}$ in $\mathcal{H}$ such that $n_{0}=n_{\text {ini }}, n_{2 \ell+1} \in N_{\text {fin }}$, and for each $i \leq \ell, L\left(H_{n_{2 i}, n_{2 i+1}}\right)$ is not empty and $\left(n_{2 i+1}, n_{2 i+2}\right)$ is in $N S C$. We can chose $n_{0}, n_{2} \ldots, n_{2 \ell}$ to be distinct according to the second item of Proposition 5.3 . In particular, $\ell$ is at most the number of nodes of $G$, and an algorithm follows. 


\section{Discussion and Related Work}

In this section, we discuss the role of the restrictions we imposed.

\subsection{Practical Motivation of Drift-boundedness}

Interpreting a node of a TC-MSC graph as specifying communications in one phase or one transaction of a distributed protocol, it is realistic to expect that in an implementation of the specification, the scenario labeling the node is performed in a bounded time, that is the difference between the date of the first and the last event of the scenario is bounded. One may thus be tempted to impose that all timed executions of the language of a TC-MSC graph are $K$-drift-bounded, for some uniform constant $K$. However, TC-MSC graphs are meant for high-level abstract description of system behaviour at an early design stage. The designer should implement flexibility in the TC-MSC graphs to adapt to different implementation hardware, and this sort of strong restriction is not acceptable. This is the reason why our $K$-drift-bounded restriction is existential in nature : we only demand that each path of a TC-MSC graph contains at least one $K$-drift-bounded timed execution, which can then be chosen in the implementation process. Notice that being $K$-drift-bounded implies to be also $\widehat{K}$-drift-bounded for $\widehat{K} \geq K$, which can then adapt to many different hardware. Conversely, a TC-MSC graph which is not $K$-drift-bounded for any $K$ have executions that require unbounded delay to finish scenarios, which is then not implementable.

Non regular examples : every TC-MSC graph used as example in this paper is $K$-drift-bounded for some small $K(K \leq 3)$, and its timed execution language is not regular. For instance, consider $G_{1}$ from Figure 1 . No assumption is made on the speed of $r$ (the constraint for $r$ is $\perp$ in the loop), which may delay message reception for an unbounded amount of time. Hence, at a given moment, there might be an arbitrary number of messages transiting from $q$ to $r$. Note that even if process $r$ is allowed to drift from $q$, the specification still allows behaviors in which they send and consume messages at the same pace. Hence unbounded drifting can occur, but can also be avoided.

Why it is necessary but not sufficient : First, we remark that the undecidability proof from [10] uses a family of TC-MSC graphs $\left(G_{i}\right)_{i \in \mathbb{N}}$ which is not $K$-drift-bounded for any $K$. Second, drift-boundedness is not sufficient to obtain a regular set of representatives. For instance, the set $L_{K}\left(G_{2}\right)$ of representatives for $G_{2}$ (see Figure 2) is not regular for any $K \geq 0$. Indeed, consider the set of timed executions where every event is executed at time 0 . This set is not regular as the number of messages from $p$ to $q$ and from $r$ to $s$ should be the same. Also, this set is included in $L_{K}\left(G_{2}\right)$. Thus, the untimed projection of $L_{K}\left(G_{2}\right)$ and of this set are equal, and $L_{K}\left(G_{2}\right)$ is not regular.

\subsection{Practical motivation of positively constrained transi- tions}

Consider a modified version of the TC-MSC graph $G_{2}$ of Figure 2 , denoted $G_{2}^{\prime}$, where constraints $[0,0]$ have been added to all messages and on all processes in the unique transition. Clearly, this enforces all the events to be done exactly at date 0 : for all $k$, all the timed executions of the TC-MSC associated with 
the path $\rho=\left(n_{0}\right)^{k}$ of $G_{2}^{\prime}$ have more than $k$ events per unit of time. Thus, this specification is not implementable as it forces an unbounded number of events to be done in 1 unit of time. Hence, it makes sense to disallow such constraints $[0,0]$ by requiring the system to be scenario-connected.

Related Work : The usual notion of strongly non-Zeno timed automaton [5] requires that there exists a bound $K^{\prime}$ such that all timed executions have at most $K^{\prime}$ events per unit of time. This imposes a given pace at which events can be done, which is very restrictive for a high-level specification. In comparison, positively constrained does not impose a pace and allows these kinds of executions in general, but implies that there exists at least one well-behaved execution for each path. For example, taking the positively constrained TC-MSC graph $G_{2}$ of Figure 2, it is possible for all the events to happen at date 0 .

Why it is not sufficient : Notice that we can slightly change the TC-MSC graph used in the undecidability proof of [10] to make it positively constrained, hence positively constrained alone does not suffice to get decidability, and a restriction such as drift boundedness is required. In fact, in this paper, we have shown that being both $K$-drift-bounded and positively constrained is sufficient to get decidability.

\subsection{Scenario-connected TC-MSC graphs}

The motivation behind the scenario-connected restriction is simple. Theorem 4.14.2 do not hold if we remove this restriction. It suffices to consider the TC-MSC graph $G_{3}$ of Figure 2 which is not scenario-connected. The timed executions $(p ! q, 1)(q ? p, 2) \cdots(p ! q, t+1)(q ? p, t+2)(s ! r, t+3)(r ? s, t+4) \cdots(s ! r, 2 t+$ $3)(r ? s, 2 t+4)$ is in $L_{1,1}\left(G_{3}\right)$ for all date $t$, and thus $L_{1,1}\left(G_{3}\right)$ is not a regular set. While we can tweak the definition of $L_{K, K^{\prime}}(G)$ to make it a regular set of representatives for $G$, this set is no longer easily defined. On the other hand, the scenario-connected restriction can be more elegantly lifted directly on Theorem 4.3 .

\subsection{Regular Set of Representatives and existential bounds on communications}

The method of regular set of representatives has been used in [11, 8] with the fixed set of existentially bounded executions as representatives. Indeed, if a language has a regular set of representatives, then it must be existentally $B$ bounded for some $B$, that is every execution is equivalent with (is a linearization of the same MSC as) a $B$ bounded execution (an execution where the number of messages sent and not yet received is at most $B$ ). In particular, if $G$ is wellformed and scenario-connected, $L_{K, K^{\prime}}(G)$ is existentally-bounded. However, the set of existentially $B$ bounded linearizations is not regular in general, even for untimed MSC-graphs. In [11, 8], another restriction on communication is used, namely globally cooperative MSC-graphs [11, which require (as regularity) an exponential blow up in the size of the MSC-graph. We have shown the versatility of the technique by proposing a new set of representatives which refines the set of existentially $B$ bounded executions, and which does not need globally cooperative MSC-graphs. We believe that this method could be used in other context than (Timed Constrained) MSC-graphs. 


\section{Conclusion}

In this paper, we have proved decidability of the language emptiness problem for a subclass of TC-MSC graphs. This problem was known to be decidable for regular TC-MSC graphs. The subclass considered in this paper contains nonregular specifications. It is characterized in terms of bounds on the time a basic scenario takes, and disallows the constraint $[0,0]$ on transitions. We believe that these two requirements do not impair implementability, and meet what designers have in mind when designing a TC-MSC : event execution takes time, and the specification is split in phases. Several related problems remain to be tackled. Given a TC-MSC graph $G$ and an integer $K$, determine whether $G$ is $K$-drift-bounded. It turns out that this problem is decidable, but its proof uses very different techniques which will be dealt with in a forthcoming paper. Also, while drift boundedness is needed to obtain decidability, we doubt that positively constrained transitions are necessary.

\section{Références}

[1] S. Akshay, M. Mukund, and K. Narayan Kumar. Checking coverage for infinite collections of timed scenarios. In CONCUR'O\%, pages 181-196, 2007.

[2] S. Akshay, P. Gastin, K. Narayan Kumar, and M. Mukund. Model checking time-constrained scenario-based specifications. In FSTTCS'10, 2010.

[3] R. Alur and D. L. Dill. A theory of timed automata. TCS, 126(2) :183-235, 1994.

[4] R. Alur and M. Yannakakis. Model checking of message sequence charts. In CONCUR'99, volume 1664 of $L N C S$, pages 114-129, 1999.

[5] E. Asarin, O. Maler, A.Pnueli, and J. Sifakis. Controller synthesis for timed automata. In IFAC Symposium on System Structure and Control, pages 469-474, 1998.

[6] P. Bouyer, S. Haddad, and P.-A. Reynier. Timed unfoldings for networks of timed automata. In ATVA'06, volume 4218 of LNCS, 2006.

[7] F. Cassez, Th. Chatain, and C. Jard. Symbolic unfoldings for networks of timed automata. In ATVA'06, volume 4218 of LNCS, pages 307-321, 2006.

[8] Ph. Darondeau, B. Genest, and L. Hélouët. Products of message sequence charts. In FOSSACS'08, volume 4962 of $L N C S$, pages 459-474, 2008.

[9] C. Dima and R. Lanotte. Distributed time-asynchronous automata. In ICTAC'07, pages 185-200, 2007.

[10] P. Gastin, K. Narayan Kumar, and M. Mukund. Reachability and boundedness in time-constrained MSC graphs. Perspectives in Concurrency Theory, 2008.

[11] B. Genest, D. Kuske, and A. Muscholl. A Kleene theorem and model checking algorithms for existentially bounded communicating automata. Inf. and Comp., 204(6) :920-956, 2006.

[12] J. G. Henriksen, M. Mukund, K. N. Kumar, M. Sohoni, and P. S. Thiagarajan. A theory of regular MSC languages. Inf. and Comp., 202(1) :1-38, 2005. 
[13] ITU-TS Recommendation Z.120 : Message Sequence Chart 1999 (MSC99), 1999.

[14] D. Lugiez, P. Niebert, and S. Zennou. A partial order semantics approach to the clock explosion problem of timed automata. TCS, 345(1) :27-59, 2005.

[15] P. Madhusudan and B. Meenakshi. Beyond message sequence graphs. In FSTTCS, pages 256-267, 2001.

[16] A. Muscholl and D. Peled. Message sequence graphs and decision problems on mazurkiewicz traces. In $M F C S^{\prime} 99$, volume 1672 of $L N C S$, pages 81-91, 1999. 


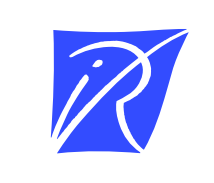

Centre de recherche INRIA Rennes - Bretagne Atlantique

IRISA, Campus universitaire de Beaulieu - 35042 Rennes Cedex (France)

Centre de recherche INRIA Futurs : Parc Orsay Université - ZAC des Vignes

4, rue Jacques Monod - 91893 ORSAY Cedex

Centre de recherche INRIA Nancy - Grand Est : LORIA, Technopôle de Nancy-Brabois - Campus scientifique

615, rue du Jardin Botanique - BP 101 - 54602 Villers-lès-Nancy Cedex

Centre de recherche INRIA Grenoble - Rhône-Alpes : 655, avenue de l'Europe - 38334 Montbonnot Saint-Ismier

Centre de recherche INRIA Paris - Rocquencourt : Domaine de Voluceau - Rocquencourt - BP 105 - 78153 Le Chesnay Cedex

Centre de recherche INRIA Sophia Antipolis - Méditerranée : 2004, route des Lucioles - BP 93 - 06902 Sophia Antipolis Cedex 